EPJ Web of Conferences 28, 10002 (2012)

DOI: $10.1051 /$ epjconf/20122810002

C Owned by the authors, published by EDP Sciences, 2012

\title{
Higgs Status and Combination
}

\author{
Gigi Rolandi on behalf of the ATLAS, CDF, CMS and D0 Collaborations \\ CERN, Geneva, Switzerland and Scuola Normale Superiore, Pisa, Italy
}

\begin{abstract}
The first combination of the exclusion limits of ATLAS and CMS for the production of a Standard Model Higgs boson is presented. The combined analyses correspond to an integrated luminosity between 1 and $2.3 \mathrm{fb}^{-1}$, depending on the channel. Using also the exclusion limits measured at LEP and at the Tevatron, the Standard Model Higgs Boson is excluded in the mass ranges $0-114.4 \mathrm{GeV}$ and $141-476 \mathrm{GeV}$ at $95 \%$ Confidence Level.
\end{abstract}

\section{Introduction and Disclaimer}

The scope of this talk is the summary and the comparison of the analyses for the search of the Higgs Boson presented at this conference by CDF and D0 [1-3] based on less than $8.6 \mathrm{fb}^{-1}$ per experiment, and by ATLAS [4-6] and CMS [8-9] based on less than $2.3 \mathrm{fb}^{-1}$ per experiment. The ATLAS[10] and CMS[11] combination [12] is presented for the first time. However, at the time of writing these proceedings, ATLAS and CMS have already presented new results based on the full 2011 LHC statistics of $4.9 \mathrm{fb}^{-1}$ [13-14]. These new results supersede the results presented here and give more stringent constraints on the mass of the Higgs Boson: approximately a low mass Higgs Boson in the Standard Model is limited in the range $115.5<\mathrm{m}_{\mathrm{H}}<127 \mathrm{GeV}$ at $95 \%$ C.L.

\section{LEP limit}

LEP analyses [15] have set a very stringent lower limit on the mass of the Standard Model Higgs Boson of 114.4 $\mathrm{GeV}$ at $95 \%$ C.L. Since the cross section increases rapidly with decreasing the mass near the kinematical limit, the $99 \%$ C.L. limit of LEP is $114.0 \mathrm{GeV}$.

\section{Tevatron limits}

The combination of CDF and D0 done with an integrated luminosity up $8.6 \mathrm{fb}^{-1}$ excludes for the Standard Model Higgs Boson the mass region between 156 and $177 \mathrm{GeV}$. This region is similar to the expected exclusion range of 148-181 GeV. The Tevatron analyses have also sensitivity in the region below the LEP exclusion and are able to exclude independently the region between 100 and $109 \mathrm{GeV}$.

\section{LHC limits}

The ATLAS and CMS Higgs limits presented at the 2011 Summer Conferences have been combined here for the first time. The combination is based on documented and public results, done with a consistent treatment of the systematic errors in the two Collaborations and with a careful and conservative attention to the correlations. The 15 independent channels entering in the combination are listed in Table 1.

Table 1. Channels entering the combination

\begin{tabular}{|c|c|c|c|}
\hline \multirow{2}{*}{ Channel } & Coll. & range $(\mathbf{G e V})$ & Lumi $\left(\mathbf{f b}^{-1}\right)$ \\
\hline \multirow{2}{*}{$\mathrm{H} \rightarrow \mathrm{bb}$} & ATLAS & $\mathbf{1 1 0 - 1 3 0}$ & 1.0 \\
& CMS & $\mathbf{1 1 0 - 1 3 5}$ & 1.1 \\
\hline \multirow{2}{*}{$\mathrm{H} \rightarrow \tau \tau$} & ATLAS & $\mathbf{1 1 0 - 1 5 0}$ & 1.1 \\
& CMS & $\mathbf{1 1 0 - 1 4 0}$ & 1.6 \\
\hline \multirow{2}{*}{$\mathrm{H} \rightarrow \gamma \gamma$} & ATLAS & $\mathbf{1 1 0 - 1 5 0}$ & 1.1 \\
& CMS & $\mathbf{1 1 0 - 1 5 0}$ & 1.7 \\
\hline \multirow{2}{*}{$\mathrm{H} \rightarrow \mathrm{WW} \rightarrow 212 v$} & ATLAS & $\mathbf{1 1 0 - 3 0 0}$ & 1.7 \\
& CMS & $\mathbf{1 1 0 - 6 0 0}$ & 1.5 \\
\hline \multirow{2}{*}{$\mathrm{H} \rightarrow \mathrm{ZZ} \rightarrow 41$} & ATLAS & $\mathbf{1 1 0 - 6 0 0}$ & 2.3 \\
& CMS & $\mathbf{1 1 0 - 6 0 0}$ & 1.7 \\
\hline $\mathrm{H} \rightarrow \mathrm{ZZ} \rightarrow 212 \tau$ & CMS & $\mathbf{1 8 0 - 6 0 0}$ & 1.1 \\
\hline \multirow{2}{*}{$\mathrm{H} \rightarrow \mathrm{ZZ} \rightarrow 212 \mathrm{q}$} & ATLAS & $\mathbf{2 0 0 - 6 0 0}$ & 1.0 \\
\multirow{2}{*}{$\mathrm{H} \rightarrow \mathrm{ZZ} \rightarrow 212 v$} & CMS & $\mathbf{2 2 5 - 6 0 0}$ & 1.6 \\
& CMS & $\mathbf{2 0 0 - 6 0 0}$ & 2.0 \\
& \multicolumn{3}{|c|}{} \\
\hline \multicolumn{2}{|c|}{}
\end{tabular}

The channels are combined following the prescription described in [10] that assumes Higgs Standard Model branching ratios. The sensitivity of each analysis is a function of the tested Higgs mass hypothesis. The weight of each analysis as a function of the mass can be computed as described in [16] and is shown in Figure 1. At high mass the $\mathrm{ZZ}$ channels give the large sensitivity, especially the final state with leptons and missing energy. 
At very low mass the $\gamma \gamma$ channel dominates. The WW channel and the $\mathrm{ZZ}$ to 4 leptons have sensitivity at low and high mass.

Figure 1: Weight of the different channels in the combined limit as function of the Higgs Boson mass

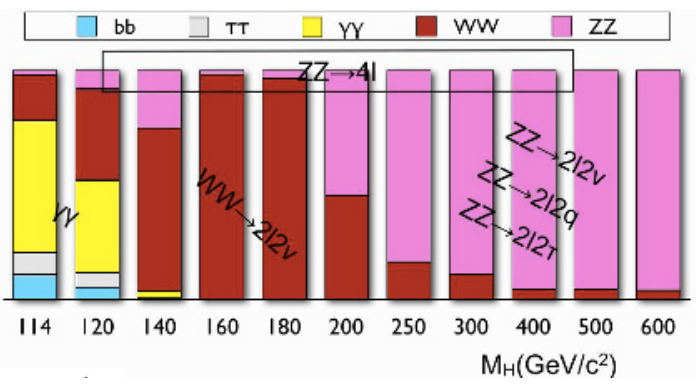

The limit on the Standard Model Higgs cross section is expressed in terms of so called "signal strength modifier " $\mu=\sigma / \sigma_{\mathrm{SM}}$, the measured cross section expressed in units of the Standard Model cross section. Figure 2 shows the value of $\mu$ that can be excluded at $95 \%$ CL by the ATLAS and CMS combination as a function of the Higgs mass. When $\mu$ is below 1 , the production of a Standard Model Higgs Boson of that mass is excluded.

Figure 2: 95\% CL upper limits of the signal strength modifier $\mu$ obtained with the CLs method

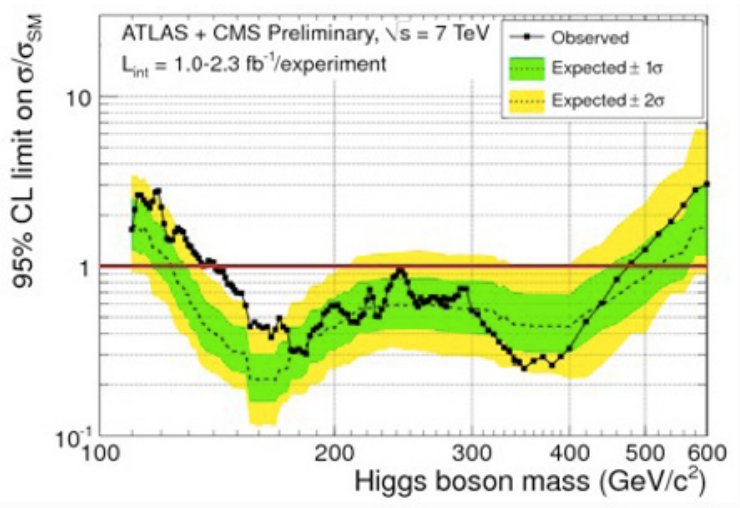

In this figure the dotted line is the expected limit in assuming there is no Higgs Boson at that given mass. The green and yellow bands give the 1 and 2 sigma expected statistical fluctuation of the expected limit. The mass range between 141 and $476 \mathrm{GeV}$ is excluded at $95 \% \mathrm{CL}$, while the expected exclusion range is between 124 and $520 \mathrm{GeV}$.

At low mass, for a large range of masses, the observed limit is above the 2 sigma band. However there is large correlation between nearby points because of the large sensitivity of the WW channel that has poor mass resolution: the same events contribute to the exclusion in a large range.

The broad excess seen at low masses is better displayed in Fig 3 that is a low masses zoom of Figure 2. The excess is largely due to the WW channel with a modulation induced by the $\mathrm{ZZ} \rightarrow 41$ and $\gamma \gamma$ channels.
Figure 3: 95\% CL upper limits of the signal strength modifier $\mu$ obtained with the CLs method

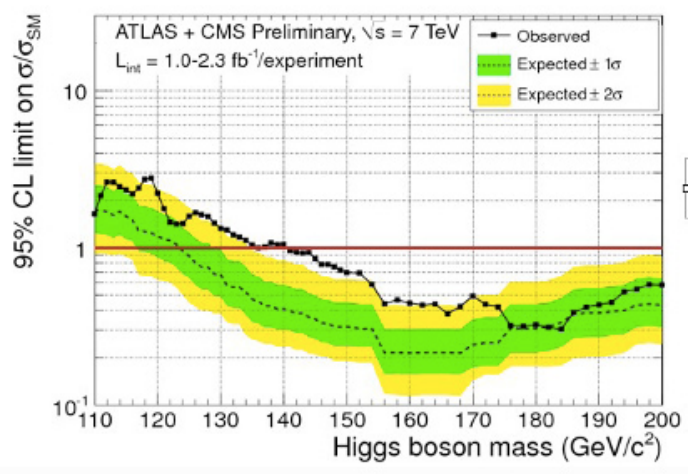

The theoretical systematic uncertainties are small, thanks to the advances in theory and to the work of the LHC Higgs cross-section group (see [12] for the long list of references). They change the expected limit by $1 \mathrm{GeV}$ at low mass and by $20 \mathrm{GeV}$ at high mass.

The statistical significance of the excess can be quantified in term of the p-value, the probability that at a given mass the background fluctuates more than what observed in the data. However this probability must be corrected for the so-called Look Elsewhere Effect (LEE) that is especially important for the channels with good mass resolution like $\gamma \gamma$ and 4 leptons. Another complication is due to the fact that at this level of integrated luminosity the number of expected background events in 4 leptons per bin of mass resolution is less than 0.5 . This reflects the good mass resolution, however the observation of a candidate in a given bin appears (statistically) unusual. Once corrected for the LEE the largest fluctuation seen in the combined data has a significance smaller than 1.6 standard deviations.

Figure 4 shows in the same plot the 95\% CL limits on $\mu$ by LHC and the Tevatron. In the non-excluded region both colliders show excess compared to the expectation. Tevatron observed $95 \%$ limit is in the $1 \sigma$ band. LHC excess has a max significance of 1.6 standard deviations. Every discovery starts with the inability to exclude, it is good to see that we have excess compared to expectation! However here we do not have the clear picture of why do we have this excess. More data (that we have already) will tell us more about the WW excess. And the other channels will become more and more sensitive.

Figure 4: 95\% CL upper limits of the signal strength modifier $\mu$ obtained with the CLs method

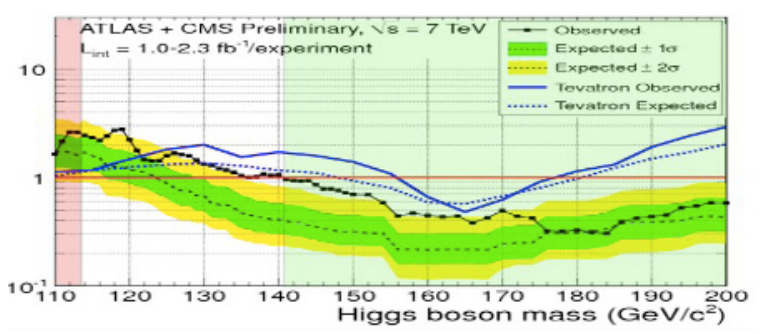


To quantify visually the impact of the LHC data on the Standard Model Higgs searches one can compare in Figure 5 and 6 the so-called green-band plot showing the measured value of the $\mathrm{W}$ and top masses vs the region allowed in the Standard Model. Before and after the LHC data.

Figure 5: Region (green) allowed by direct Higgs searches in the Standard Model as a function of the W, and top masses compared with direct measurements. Before LHC results.

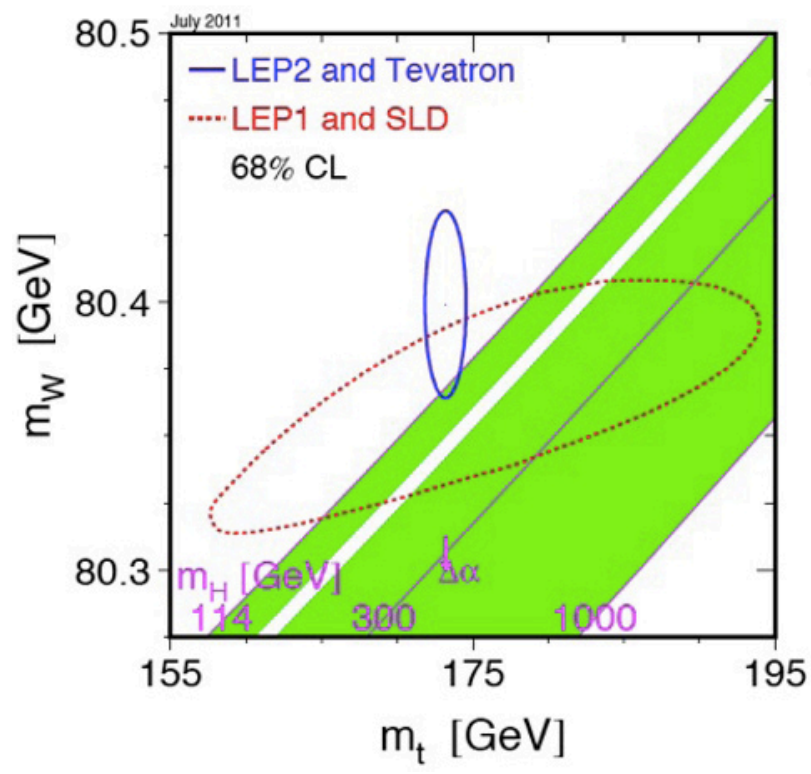

Figure 6: Region (green) allowed by direct Higgs searches in the Standard Model as a function of the W, and top masses compared with direct measurements. After LHC results

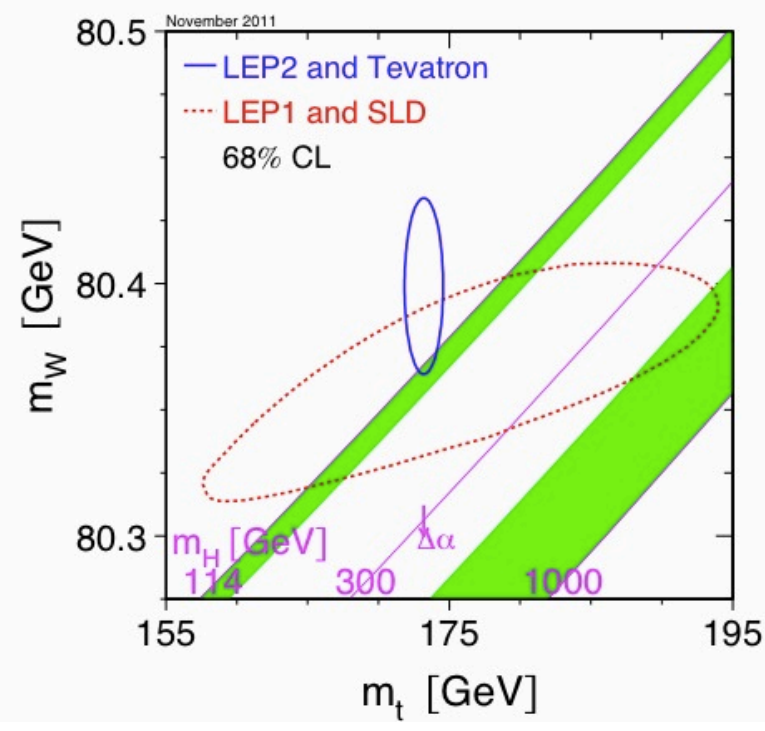

Little room is left for the SM Higgs : $114<\mathrm{m}_{\mathrm{H}}<141$ GeV@ 95\% CL. ATLAS and CMS will analyze the x3 data already collected before 2012 Winter Conferences.

CDF and D0 will provide the final results on $10 \mathrm{fb}^{-1}$ by the 2012 Summer Conferences. On the same time scale a combination $\mathrm{LHC}+$ Tevatron is expected.

\section{References}

1. K. Petridis, "Searches at 'high' Higgs mass @ Tevatron", these proceedings

2. F. Sforza, "Searches for low mass Higgs @ Tevatron", these proceeding

3. W. Yao, "Standard Model Higgs Combination @ Tevatron", these proceedings

4. L. Iconomidou-Fayard, "Searches for $\mathrm{H}->\mathrm{WW}, \mathrm{ZZ}$ (a) ATLAS", these proceedings

5. M. Duehrssen, "Searches for Low mass Higgs @ ATLAS", these proceedings

6. F. Tarrade, "Standard Model Higgs Combination @ ATLAS", these proceedings

7. G. Codispoti, "Searches for H -> WW, ZZ @ CMS", these proceedings

8. V. Rekovic, "Searches for Low mass Higgs @ CMS", these proceedings

9. M. Bluj, "Standard Model Higgs Combination @ CMS", these proceedings

10. ATLAS Collaboration, "The ATLAS experiment at the CERN LHC", JINST 3:S08003, 2008.

11. CMS Collaboration, "The CMS experiment at the CERN LHC", JINST 3:S08004, 2008

12. ATLAS and CMS Collaborations, "Combined Standard Model Higgs boson searches with up to 2.3 $\mathrm{fb}^{-1}$ of pp collision data at $\sqrt{\mathrm{s}}=7 \mathrm{TeV}$ at the LHC". ATLAS-CONF-2011-157 and CMS PAS HIG-11-023, 2011

13. ATLAS Collaboration, "Combination of Higgs Boson Searches with up to $4.9 \mathrm{fb}-1$ of pp Collisions Data Taken at a center-of-mass energy of $7 \mathrm{TeV}$ with the ATLAS Experiment at the LHC", ATLASCONF-2011-163, 2011

14. CMS Collaboration, "Combination of SM Higgs Searches", CMS-PAS-HIG-11-032, 2011

15. R. Barate and others (LEP Working Group for Higgs boson searches and ALEPH, DELPHI, L3, and OPAL Collaborations), "Search for the standard model Higgs boson at LEP”, Phys. Lett. B565 (2003) 61-75, arXiv:hep-ex/0306033. doi:10.1016/S03702693(03)00614-2.

16. G. Cowan, K. Cranmer, E. Gross, O. Vitells, "Asymptotic formulae for likelihood-based tests of new physics”, Eur.Phys.J. C71 (2011) 1554 